\title{
Estágios em farmácia comunitária nos cursos de graduação em Farmácia no Brasil
}

Community pharmacy internships in pharmacy undergraduate programs in Brazil

\author{
A. A. Foppa ${ }^{1 *}$; A. R. Mesquita ${ }^{2}$; S. A. M. Mendonça ${ }^{2}$; C. Chemello \\ ${ }^{\text {I} P r o g r a m a ~ d e ~ P o ́ s-G r a d u a c ̧ a ̃ o ~ e m ~ M e d i c a m e n t o s ~ e ~ A s s i s t e ̂ n c i a ~ F a r m a c e ̂ u t i c a, ~ F a c u l d a d e ~ d e ~ F a r m a ́ c i a, ~ U n i v e r s i d a d e ~}$ \\ Federal de Minas Gerais, 31270-901, Belo Horizonte - Minas Gerais, Brasil \\ ${ }^{2}$ Departamento de Farmácia Social da Faculdade de Farmácia, Universidade Federal de Minas Gerais, 31270-901, \\ Belo Horizonte - Minas Gerais, Brasil \\ *alinefoppa@yahoo.com.br \\ (Recebido em 17 de fevereiro de 2021; aceito em 08 de maio de 2021)
}

\begin{abstract}
Os cursos de Farmácia no Brasil estão em um processo de mudança curricular. A aprendizagem experiencial tem um papel essencial na construção do conhecimento, entretanto há uma escassez de estudos sobre o tema. Este estudo descreve os aspectos organizacionais e pedagógicos dos estágios em farmácia comunitária dos cursos de Farmácia do Brasil. Trata-se de um estudo observacional descritivo transversal sobre os estágios em farmácia comunitária, ofertados por cursos presenciais, com início anterior a 2015 (n=288). Os dados foram coletados de documentos e questionários autoaplicáveis enviados para coordenadores e/ou docentes responsáveis pelos estágios em farmácia comunitária. Os cursos de graduação em Farmácia que responderam $(\mathrm{n}=98)$ possuíam uma mediana de 2 [1;3] estágios em farmácia comunitária, com carga horária total de 263,5 $[146,5 ; 405]$ horas, realizados em maior proporção em farmácias comunitárias privadas $(76,5 \% ; n=75)$, seguido pelas farmácias comunitárias vinculadas ao Sistema Único de Saúde $(53,1 \%$; n=52) e farmácias universitárias $(46,9 \% ; n=45)$. Quanto aos aspectos pedagógicos, os docentes visitaram os locais de estágio com mediana de $5[2 ; 18,5]$ vezes/semestre e apenas $39 \%(n=25)$ dos cursos possuíam tanto disciplinas das "ciências sociais / comportamentais / administrativas de farmácia", quanto das "ciências clínicas" como prérequisito para o estágio em farmácia comunitária. O processo de ensino-aprendizagem mais utilizado foi "estudante observa o supervisor e depois executa com 100\% de supervisão" e a ferramenta de avaliação mais empregada foram os relatórios orais/escritos $(80,6 \% ; n=79)$. O estudo revela importantes lacunas na organização dos estágios em farmácia comunitária, o que pode comprometer a formação para atuar nessa área profissional.
\end{abstract}

Palavras-chave: educação em farmácia, estágio, serviços comunitários de farmácia.

The pharmacy undergraduate programs are in curriculum change process. The experiential learning has essential role in the construction of knowledge and there is the gap in the literature about studies of this nature. This study describes organizational and pedagogical aspects of community pharmacy internships in pharmacy undergraduate programs in Brazil. This is a cross-sectional descriptive observational study on community pharmacy internships required by face-to-face pharmacy programs inaugurated before 2015 $(\mathrm{n}=288)$. Data were collected from documents and self-administered questionnaires by coordinators and/or teachers in charge of community pharmacy internships. The pharmacy programs $(\mathrm{n}=98)$ required a median of $2[1 ; 3]$ community pharmacy internships, totalizing $263.5[146.5 ; 405]$ hours, completed either in private pharmacies $(76.5 \% ; n=75)$, SUS pharmacies $(53.1 \%$; $n=52)$ or university pharmacies $(46.9 \% ; n=45)$. Regarding pedagogical aspects, teachers visit the internship sites with a median of 5 [2;18.5] times semiannually, and only $39 \% \quad(n=25)$ of the pharmacy programs require courses on both "social/behavioral/administrative sciences" and "clinical sciences" as a prerequisite for community pharmacy internships. The teaching-learning process most commonly used is "student observes preceptor and practices with supervision" and the assessment tool mostly used are oral/written reports $(80.6 \% ; n=79)$. The results reveal important organizational gaps in the internships required by pharmacy programs in Brazil, which may affect future professionals intending to work in community pharmacies.

Keywords: pharmacy education, internship, community pharmacy services.

\section{INTRODUÇÃO}

O ensino experiencial é, indiscutivelmente, uma etapa imprescindível do processo de ensinoaprendizagem do cuidado em saúde [1-5]. É por meio dele que os estudantes de Farmácia são 
preparados para assumir sua responsabilidade no cuidado ao paciente [1], visto que aprender pela experiência permite ao estudante vivenciar dilemas profissionais reais, refletir sobre o que observou na prática com profissionais experientes e construir sua identidade profissional [2]. Isso tem levado países referências na oferta de serviço farmacêutico centrado no paciente, como Canadá e Estados Unidos, a concentrar seus esforços na expansão, aprimoramento e qualificação das experiências práticas em farmácias, a luz dos novos papéis e responsabilidades dos farmacêuticos $[4,6,7]$.

No Brasil, o componente experiencial, tanto por meio da integração ensino-serviço, quanto nos serviços escola, tem sido cada vez mais valorizado e, principalmente, após a implementação das Diretrizes Curriculares Nacionais (DCN), considerado como crucial no processo educacional, sendo recomendado desde o início da jornada acadêmica [8, 9]. Preconiza-se, ainda, uma diversificação dos cenários de ensino-aprendizagem, sendo o Sistema Único de Saúde (SUS) e a farmácia universitária campos obrigatórios [10], uma vez que a formação do farmacêutico, semelhante a dos demais profissionais de saúde, deve objetivar o desenvolvimento de competências para atuar de forma resolutiva em serviços diretamente ofertados ao indivíduo, família e comunidade no âmbito do SUS [11].

O estágio obrigatório, previsto no projeto político pedagógico do curso e requisito para a obtenção de diploma, integra o itinerário formativo do educando, e deve ser realizado em ambiente de trabalho com acompanhamento efetivo do docente orientador da instituição de ensino superior e do preceptor vinculado à instituição concedente, o qual deverá possuir "formação ou experiência profissional na área de conhecimento desenvolvida no curso do estagiário". O estágio ocorre mediante a celebração de um termo de compromisso entre instituição de ensino, concedente e educando, os quais definem conjuntamente o plano de atividades do estagiário. Cabe a Instituição de Ensino Superior avaliar as instalações da parte concedente do estágio e sua adequação à formação cultural e profissional do educando; exigir do educando a apresentação periódica de relatório das atividades; e elaborar normas complementares e instrumentos de avaliação dos estágios de seus educandos [12].

Os estudos recentes relacionados ao ensino farmacêutico no Brasil têm proposto o desenvolvimento de metodologias inovadoras, em ambientes simulados, as quais tem demonstrado efetividade no treinamento da prática clínica [13-15]. No entanto, existe uma lacuna na literatura sobre o ensino em cenários reais em cursos de graduação em Farmácia no país e uma escassez e/ou falta de definição do arcabouço educacional para seu ensino-aprendizagem no cotidiano de serviços de saúde [16]. Tendo em vista que o farmacêutico está inserido predominantemente em farmácias comunitárias, fenômeno semelhante ao que acontece no restante do mundo [17, 18], o ensino deve privilegiar o desenvolvimento de competências para atuar de forma eficiente em atividades relativas a essa área, como no acesso ao medicamento e na provisão de serviços no pós-acesso [19]. Assim, entendendo a essencialidade do ensino experiencial na construção do conhecimento e a ênfase para a necessidade de formação de profissionais para atuar em farmácias comunitárias, principalmente àquelas vinculadas ao SUS, objetiva-se com este estudo descrever os aspectos organizacionais e pedagógicos dos estágios em farmácias comunitárias dos cursos de Farmácia no Brasil.

\section{MÉTODOS}

O presente estudo é parte do projeto "Proposição de um percurso formativo para o ensino do cuidado em saúde nos cursos de graduação em Farmácia do Brasil”, aprovado pelo Comitê de Ética em Pesquisa da UFMG (CAAE $n^{\circ}$ 87998718.2.0000.5149), e compõe a tese de doutorado intitulada "Educação para o cuidado em saúde nos estágios dos cursos de Farmácia: um olhar sobre o contexto comunitário". O desenho de estudo utilizado foi o observacional descritivo transversal.

\subsection{Amostragem}

Inicialmente, foi realizado um levantamento das Instituições de Ensino Superior (IES) que possuem curso de graduação em Farmácia. Esses dados foram obtidos por meio da lista de cursos do Ministério da Educação (MEC) atualizadas em julho de 2018, sendo que no decorrer da coleta os dados foram conferidos no website do MEC (e-Mec) [20] para possíveis atualizações. Foram 
considerados elegíveis os cursos de graduação em Farmácia na modalidade presencial e em atividade. Os critérios de exclusão foram: cursos com início a partir do ano de 2015 e impossibilidade de confirmação, via telefônica, do e-mail do docente responsável pelo estágio em farmácia comunitária e/ou coordenador do curso de Farmácia. Entre os principais motivos da inviabilidade de confirmação estavam: disponibilização online apenas de telefone geral da IES; recusa do fornecimento de e-mail e não atendimento do telefone após três tentativas no período da manhã, tarde e noite.

Após esse levantamento, a seleção da amostra foi realizada por conveniência, isto é, de acordo com a disponibilidade do docente responsável pelos estágios em farmácias comunitárias e/ou coordenador do curso em responder ao questionário. Os docentes que aceitaram participar do estudo preencheram eletronicamente o Termo de Consentimento Livre Esclarecido.

\subsection{Coleta de dados}

Os dados foram coletados durante os meses de novembro de 2018 a janeiro de 2019, em duas etapas: (1) elaboração e envio do questionário por e-mail aos docentes responsáveis pelos estágios em farmácias comunitárias e/ou coordenadores do curso via ferramenta online e (2) pesquisa documental.

A primeira etapa constituiu-se da elaboração do questionário (instrumento autoaplicável), realização de pré-teste e envio do instrumento aos docentes responsáveis por estágio em farmácias comunitárias e/ou coordenadores do curso de Farmácia das IES consideradas elegíveis para o estudo. A Tabela 1 descreve as variáveis do estudo.

Tabela 1: Variáveis do estudo - aspectos organizacionais e pedagógicos.

\begin{tabular}{|c|c|}
\hline $\begin{array}{l}\text { Aspectos } \\
\text { organizacionais }\end{array}$ & 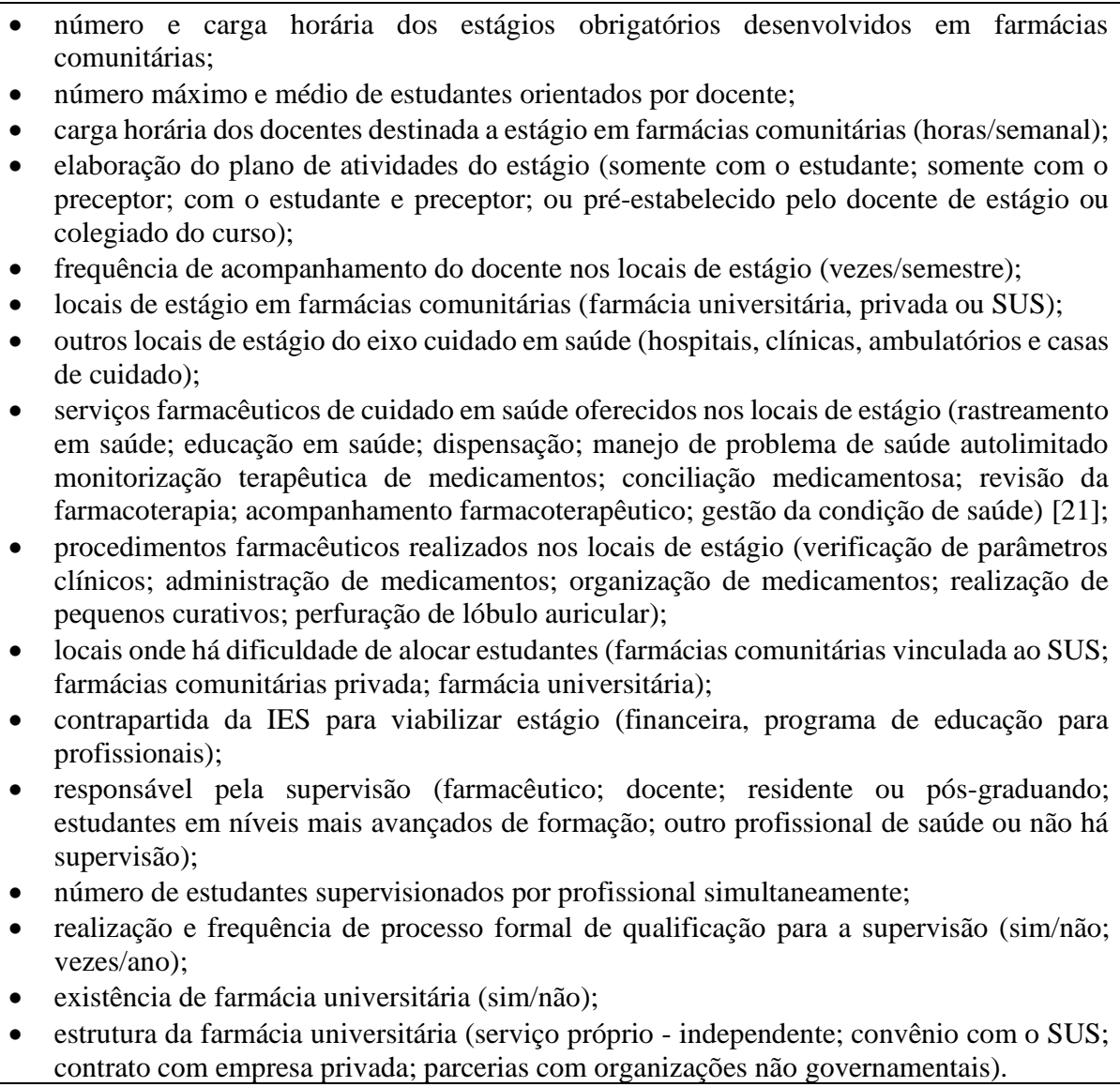 \\
\hline
\end{tabular}


Tabela 1: (continuação)

\begin{tabular}{lll}
\hline $\begin{array}{l}\text { Aspectos } \\
\text { pedagógicos }\end{array}$ & existência de disciplina pré-requisito para a realização dos estágios em farmácias \\
& comunitárias (sim/não); \\
& conteúdos categorizados de acordo com “Accreditation Council for Pharmacy Education", \\
& - ACPE (ciências biomédicas básicas; ciências farmacêuticas; ciências sociais / \\
& comportamentais / administrativas de farmácia; ciências clínicas) [22]*; \\
& processo de ensino-aprendizagem (estudante apenas observa o supervisor; estudante observa \\
& o supervisor e depois executa com 100\% de supervisão; estudante observa o supervisor e \\
& depois executa sem supervisão; estudante não observa o supervisor e executa com 100\% de \\
& supervisão; ou estudante não observa o supervisor e executa sem supervisão); \\
& instrumentos de avaliação do estágio (prova escrita; relatório; discussão nos fóruns, \\
& utilizando o Ambiente Virtual de Aprendizagem; feedback oral ou escrito, feito pelo \\
& supervisor, sobre a performance do estudante no estágio; Longbook; Objective Structured \\
& Clinical Examination - OSCE; Long-case; Mini-Clinical Evaluation Exercise - Mini-Cex; \\
& MiniPat; Patient-survey; Portfólio; Autoavaliação do estudante) [23].
\end{tabular}

* A escolha em categorizar os conteúdos segundo a classificação ACPE, ao invés da DCN/2017, ocorreu para possibilitar estudos comparativos entre a realidade brasileira e internacional.

O pré-teste, realizado antes do envio do questionário, consistiu na aplicação prévia do instrumento a quatro docentes responsáveis pelo estágio e/ou coordenadores de curso de IES privadas e públicas, das regiões sul, sudeste e nordeste. O pré-teste teve como objetivo avaliar o instrumento quanto à clareza e precisão dos termos utilizados; formato das perguntas; quantidade e ordenamento das perguntas; introdução do questionário ao participante e tempo gasto para resposta. Depois de feitos os ajustes, o instrumento foi enviado aos docentes responsáveis pelo estágio e/ou coordenador do curso de todos os cursos de Farmácia considerados elegíveis.

De modo a complementar dados coletados na etapa anterior, foi realizada pesquisa documental no site e-MEC e no sítio eletrônico de todas as IES que responderam ao instrumento. Foram coletadas informações sobre:

- IES: região do país (norte, nordeste, centro-oeste, sudeste e sul); unidade federativa; dependência administrativa (pública ou privada);

- Curso de Farmácia: ano de início de funcionamento, periodicidade (semestral ou anual); número de vagas autorizadas por ano; carga horária total do curso; turno do curso (noturno, diurno, diurno e noturno, diurno ou noturno).

\subsection{Análise de dados}

Os dados foram tabulados no software Microsoft Excel ${ }^{\circledR}$ e analisados usando-se o programa SPSS para Windows versão 25.0. As variáveis categóricas foram representadas pela frequência absoluta e relativa, e intervalo de confiança da proporção. As variáveis quantitativas foram representadas pela mediana e intervalo interquartílico (mediana [p25; p75]), juntamente com os valores mínimos e máximos. Para comparar as proporções entre os tipos de farmácias comunitárias utilizadas como campo de prática pelos cursos (farmácia universitária, farmácia privada e farmácia do SUS) foi realizado o modelo de Equações de Estimações Generalizadas (GEE Model) [24-26]. Quando significativo, o teste post-hoc de Bonferroni foi utilizado.

\section{RESULTADOS}

Dos 574 cursos de Farmácia presenciais e em atividade, segundo dados do MEC de julho de 2018, 288 cursos foram considerados elegíveis e incluídos no estudo, sendo que destes, 98 responderam ao questionário. O fluxograma da amostragem encontra-se ilustrado na Figura 1. 


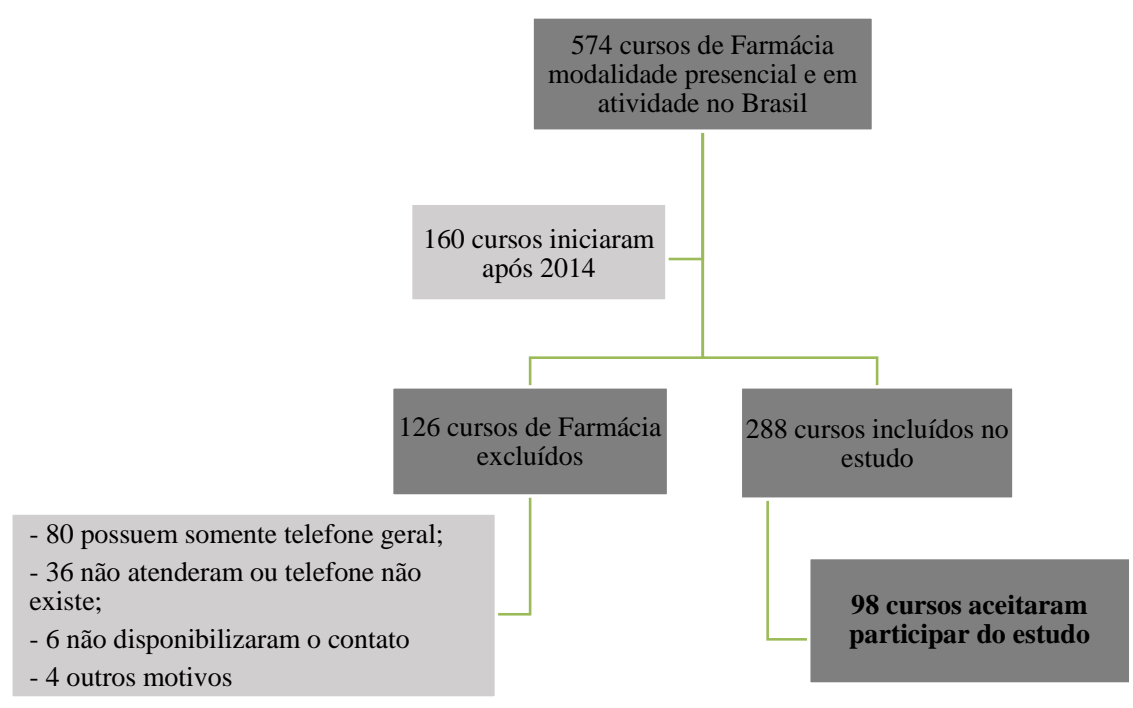

Figura 1: Fluxograma de seleção dos participantes do estudo.

Participaram do estudo IES de todas as regiões do país, prevalecendo a região sudeste $(36,7 \%$; $\mathrm{n}=36)$, seguida pela região sul $(29,6 \% ; n=29)$, nordeste $(18,4 \% ; n=18)$, centro-oeste $(10,2 \% ; n=10)$ e norte $(5,1 \% ; n=5)$. Com relação à distribuição nos estados, apenas instituições do Acre, Alagoas, Amapá, Roraima e Tocantins não participaram da pesquisa. O curso de Farmácia mais antigo iniciou em 1832 e houve um número expressivo de cursos participantes que iniciaram entre 2008 e $2009(\mathrm{n}=23)$. Na Tabela 2 encontram-se descritas as características gerais dos cursos de Farmácia participantes do estudo.

Tabela 2: Características dos cursos de Farmácia participantes do estudo (n=98).

\begin{tabular}{|c|c|}
\hline & n (\%) \\
\hline \multirow[t]{2}{*}{ Dependência administrativa } & Privada: $79(80,6)$ \\
\hline & Pública: $19(19,4)$ \\
\hline \multirow[t]{2}{*}{ Periodicidade do curso } & Semestral: $90(91,8)$ \\
\hline & Anual: $8(8,2)$ \\
\hline \multirow[t]{6}{*}{ Turno do curso } & Noturno: $20(20,4)$ \\
\hline & Diurno: $22(22,4)$ \\
\hline & Integral $*: 21(21,4)$ \\
\hline & Diurno ou noturno $* *: 20(20,4)$ \\
\hline & IES não disponibiliza informação: $15(15,3)$ \\
\hline & Mediana [p25; p75] \\
\hline Número de vagas autorizadas & $100[73 ; 160]$ \\
\hline Carga horária do curso & $4320,00[4029.7 ; 4599,7]$ \\
\hline
\end{tabular}

\subsection{Aspectos organizacionais dos estágios em farmácias comunitárias}

Os cursos de graduação em Farmácia possuíam uma mediana de 2 [1;3] estágios em farmácias comunitárias, com carga horária total de 263,5 [146,5; 405] horas, realizados em maior proporção nas farmácias comunitárias privadas $(76,5 \% ; n=75)$, seguido pelas farmácias comunitárias vinculada ao SUS $(53,1 \% ; n=52)$ e farmácias universitárias $(46,9 \% ; n=45)$. Do total das IES participantes, 53,1\% (n=52) possuíam farmácia universitária, destas, 55,8\% (n=29) eram independentes, 19,2\% $(n=10)$ conveniadas ao SUS e $15,4 \%(n=8)$ a empresas privadas. Na Tabela 3 encontram-se sumarizadas as características gerais dos estágios em farmácias comunitárias. 
Tabela 3: Características dos estágios obrigatórios em farmácias comunitárias - geral (n=98).

\begin{tabular}{lcc}
\hline & \multicolumn{1}{c}{ Mediana [p25; p75] } & Min-Máx \\
\hline Número de estágios & $2[1 ; 3]$ & $0-5$ \\
Carga horária total dos estágios & $263,5[146,5 ; 405]$ & $0-975$ \\
Número máximo de estudantes orientados por docente & $10[6 ; 10]$ & $1-100$ \\
Número médio de estudantes orientados por docente & $8[5 ; 10]$ & $1-100$ \\
Carga horária semanal dos docentes (em horas) & $8[4 ; 20]$ & $0 ; 40$ \\
Número de vezes que o docente vai ao local de estágio & $5[2 ; 18,5]$ & \multicolumn{1}{c}{ n (\%) } \\
(vezes/semestre) & \multicolumn{1}{c}{ Realizada com o estudante e preceptor: 13 (13,3) } \\
\cline { 2 - 3 } Elaboração do plano de atividades do estágio & Realizada somente com o preceptor: 9 (9,2) \\
\cline { 2 - 3 } & Pré-estabelecido pelo docente ou colegiado do curso: \\
Locais de estágio & 76 (77,6) \\
& Farmácia universitária: 46 (46,9) \\
& Farmácia privada: 75 (76,5) \\
& Farmácia vinculada ao SUS: 52 (53,1) \\
\hline
\end{tabular}

Nas farmácias comunitárias vinculadas ao SUS, a supervisão era feita majoritariamente por farmacêuticos $(68,8 \%$; $n=53)$, seguido por docentes, $(26 \% ; n=20)$, sendo que em uma IES esta função ficava a cargo de outro profissional. Nas farmácias universitárias, os farmacêuticos (53,2\%; $\mathrm{n}=25)$ e docentes $(44,7 \%$; $\mathrm{n}=21)$ apresentaram uma proporção semelhante de responsabilidade pela supervisão, com uma mediana de $5[3,75 ; 8]$ estudantes por profissional simultaneamente. Com relação à qualificação formal, nas farmácias universitárias os profissionais responsáveis pela supervisão recebiam qualificação em uma maior proporção de IES $(50 \% ; n=49)$, com uma mediana de 2 [2;2] vezes ao ano, quando comparada às farmácias vinculadas ao SUS $(33,3 \% ; n=26), 2[1 ; 4]$ vezes ao ano, e às farmácias privadas $(29,3 \% ; n=22), 1,5[1 ; 2]$ vezes ao ano.

Os cursos de Farmácia apresentaram dificuldade em alocar seus estudantes nos estágios em farmácia comunitária, principalmente nas vinculadas ao SUS $(48 \% ; n=47)$, seguida pelas farmácias privadas $(29,6 \% ; n=29)$ e farmácias universitárias $(21,4 \% ; n=21)$, sendo que apenas $33,7 \%(n=33)$ não apresentavam nenhuma dificuldade. Para viabilizar os estágios, 24,5\% ( $n=24)$ e $7,1 \%(n=7)$ das IES ofereciam programa de educação para farmacêuticos do SUS e vinculados à farmácia privada, respectivamente; $19,4 \%(\mathrm{n}=19)$ contrapartida financeira para farmácia privada; e $58,2 \%$ ( $n=57$ ) não oferece nenhuma contrapartida.

Considerando os serviços de cuidado farmacêutico oferecidos nos locais de estágio, verificouse que a farmácia universitária foi a que mais os ofertou (Tabela 4). A revisão da farmacoterapia $(\mathrm{p}=0,002)$ e acompanhamento farmacoterapêutico $(\mathrm{p}<0,001)$ foram ofertados em maior proporção pelas farmácias universitárias, quando comparadas com as farmácias privadas e às vinculadas ao SUS; já a educação em saúde foi maior apenas quando comparada à farmácia privada $(78,8 \%$ versus $52 \%$; $\mathrm{p}=0,004)$; e o manejo de problema de saúde autolimitado foi maior quando comparado a farmácias vinculadas ao SUS $(46,2 \%$ versus $16,7 \%$; $=0,001)$. As farmácias privadas realizavam mais alguns procedimentos farmacêuticos quando comparadas à farmácia universitária: verificação de parâmetros clínicos $(88 \%$ versus $75 \%$; $\mathrm{p}=0,045)$ e perfuração de lóbulo auricular (37,3 versus $17,3 \% ; \mathrm{p}=0,009$ ) (Tabela 4). Esses procedimentos não foram analisados em farmácias vinculadas ao SUS, uma vez que em tais locais estes são realizados pela equipe de enfermagem.

Além das farmácias comunitárias, 92,9 \% (n=91) das IES contavam com outros locais para a realização de estágios vinculados ao cuidado em saúde. Entre os principais locais estavam: hospitais (98,9\%; $n=90)$, clínicas $(35,2 \% ; n=32)$, ambulatórios $(27,5 \% ; n=25)$ e casas de cuidado $(14,3 \%$; $\mathrm{n}=13)$. No entanto, nenhum dos serviços farmacêuticos oferecidos diretamente ao paciente nesses locais apresentou maior proporção quando comparado à farmácia universitária, no entanto, a conciliação medicamentosa apresentou maior proporção nesses locais quando comparada à farmácia privada e a farmácia vinculada ao SUS $(\mathrm{p}<0,001)$. 
Tabela 4: Comparação entre os serviços e procedimentos farmacêuticos realizados nos locais de estágio em farmácias comunitárias.

\begin{tabular}{|c|c|c|c|c|c|c|c|c|}
\hline & & \multicolumn{2}{|c|}{ universitária } & \multicolumn{2}{|r|}{ privada } & \multicolumn{2}{|r|}{ SUS } & \multirow[t]{2}{*}{$\mathrm{P}$} \\
\hline & & $\mathrm{n}$ & $\%[$ IC95\%] & $\mathrm{n}$ & $\%[\mathrm{IC} 95 \%]$ & $\mathrm{n}$ & $\%[$ IC95\%] & \\
\hline Serviços & Rastreamento em saúde & 20 & $38,5[26,2 ; 52]$ & 20 & $26,7[17,7 ; 37,4]$ & 20 & $25,6[17 ; 36,1]$ & 0,230 \\
\hline \multirow[t]{8}{*}{ farmacêuticos } & Educação em saúde & 41 & $78,8 \mathrm{a}[66,4 ; 88,2]$ & 39 & $52,0 \mathrm{~b}[40,8 ; 63,1]$ & 52 & $66,7 \mathrm{ab}[55,7 ; 76,4]$ & 0,004 \\
\hline & Dispensação & 47 & $90,4[80,2 ; 96,2]$ & 69 & $92[84,3 ; 96,6]$ & 69 & $88,5[80 ; 94,1]$ & 0,755 \\
\hline & Manejo problema de saúde autolimitado & 24 & $46,2 \mathrm{a}[33,1 ; 59,6]$ & 25 & $33,3 \mathrm{ab}[23,5 ; 44,5]$ & 13 & $16,7 \mathrm{~b}[9,7 ; 26,1]$ & 0,001 \\
\hline & Monitorização terapêutica de medicação & 0 & $0[0 ; 0]$ & 13 & $25[14,8 ; 37,9]$ & 0 & $0[0 ; 0]$ & - \\
\hline & Conciliação medicamentosa & 18 & $34,6[22,8 ; 48,1]$ & 19 & $25,3[16,6 ; 36]$ & 14 & $17,9[10,7 ; 27,5]$ & 0,146 \\
\hline & Revisão da farmacoterapia & 32 & $61,5 \mathrm{a}[48 ; 73,8]$ & 30 & $40,0 \mathrm{~b}[29,5 ; 51,3]$ & 27 & $34,6 \mathrm{~b}[24,8 ; 45,6]$ & 0,002 \\
\hline & Acompanhamento farmacoterapêutico & 34 & $65,4 \mathrm{a}[51,9 ; 77,2]$ & 24 & $32,0 \mathrm{~b}[22,3 ; 43,1]$ & 30 & $38,5 b[28,2 ; 49,5]$ & $<0,001$ \\
\hline & Gestão da condição de saúde & 11 & $21,2[11,8 ; 33,6]$ & 9 & $12[6,1 ; 20,8]$ & 15 & $19,2[11,7 ; 29]$ & 0,365 \\
\hline \multirow{5}{*}{$\begin{array}{l}\text { Procedimentos } \\
\text { farmacêuticos }\end{array}$} & Verificação de parâmetros clínicos & 39 & $75,0[62,1 ; 85,2]$ & 66 & $88,0[79,2 ; 93,9]$ & & & 0,043 \\
\hline & Administração de medicamentos & 20 & $38,5[26,2 ; 52]$ & 38 & $50,7[39,5 ; 61,8]$ & & & 0,120 \\
\hline & Organização de medicamentos & 40 & $76,9[64,2 ; 86,7]$ & 59 & $78,7[68,4 ; 86,7]$ & & & 0,813 \\
\hline & Realização de pequenos curativos & 8 & $15,4[7,6 ; 26,9]$ & 11 & $14,7[8,1 ; 23,9]$ & & & 0,898 \\
\hline & Perfuração de lóbulo auricular & 9 & $17,3[8,9 ; 29,2]$ & 28 & $37,3[27 ; 48,6]$ & & & 0,009 \\
\hline
\end{tabular}

Modelo Equações de Estimações Generalizadas; Letras distintas representam categorias com distribuições estatisticamente distintas. 


\subsection{Aspectos pedagógicos dos estágios em farmácias comunitárias}

Do total de IES participantes, $65,3 \%$ (n=64) possuíam disciplinas pré-requisito para a realização dos estágios em farmácias comunitárias. Destas, 65,6\% (n=42) afirmaram que tais disciplinas pertenciam à categoria "ciências sociais / comportamentais / administrativas de farmácia", 60,9\% $(n=39)$ a "ciências clínicas", 54,7\% (n=35) a "ciências farmacêuticas" e 15,6\% $(n=10)$ a "ciências biomédicas básicas". Ressalta-se que apenas 39\% (n=25) das IES possuíam tanto disciplinas das "ciências sociais / comportamentais / administrativas de farmácia", quanto das "ciências clínicas" como pré-requisito para o estágio em farmácias comunitárias, sendo que $12,6 \%$ (n=8) não possuía nenhuma disciplina dessas duas categorias como pré-requisito para o estágio em farmácias comunitárias.

O processo de ensino-aprendizagem utilizado com maior frequência em todos os ambientes de estágio em farmácias comunitárias foi "estudante observa o supervisor e depois executa com $100 \%$ de supervisão". Verificou-se um número considerável em que o estudante executava as atividades sem supervisão, com maior proporção nas farmácias vinculadas ao SUS; e havia um maior desconhecimento por parte do docente sobre o processo de ensino-aprendizagem nas farmácias privadas. Na Tabela 5 está descrito o processo de ensino-aprendizagem utilizado nos locais de estágio em farmácias comunitárias. 
Tabela 5: Comparação entre farmácias para cada processo de ensino-aprendizagem.

\begin{tabular}{|c|c|c|c|c|c|c|c|}
\hline & \multicolumn{2}{|r|}{ universitária } & \multicolumn{2}{|r|}{ privada } & \multicolumn{2}{|r|}{ SUS } & \multirow[t]{2}{*}{$\mathrm{P}$} \\
\hline & $\mathrm{n}$ & $\%[$ [C95\%] & $\mathrm{n}$ & $\%$ [IC95\%] & $\mathrm{n}$ & $\%$ [IC95\%] & \\
\hline Estudante apenas observa o supervisor & 0 & $0[0 ; 0]$ & 4 & $5,3[1,8 ; 12,2]$ & 4 & $5,1[1,8 ; 11,7]$ & 0,956 \\
\hline $\begin{array}{l}\text { Estudante não observa executa com } \\
\text { supervisão }\end{array}$ & 0 & $0[0 ; 0]$ & 3 & $4,0[1,1 ; 10,3]$ & 0 & $0[0 ; 0]$ & - \\
\hline $\begin{array}{l}\text { Estudante não observa executa sem } \\
\text { supervisão }\end{array}$ & 0 & $0[0 ; 0]$ & 1 & $1,3[0,1 ; 6,1]$ & 0 & $0[0 ; 0]$ & - \\
\hline $\begin{array}{l}\text { Estudante observa e executa com } \\
\text { supervisão }\end{array}$ & 44 & $95,7 \mathrm{a}[85,8 ; 99,1]$ & 57 & $76,0 \mathrm{c}[65,5 ; 84,6]$ & 63 & $80,8 \mathrm{~b}[70,9 ; 88,3]$ & $\mathbf{0 , 0 1 3}$ \\
\hline $\begin{array}{l}\text { Estudante observa e executa sem } \\
\text { supervisão }\end{array}$ & 2 & $4,3[0,9 ; 13,2]$ & 5 & $6,7[2,6 ; 14]$ & 9 & $11,5[5,9 ; 20]$ & 0,161 \\
\hline Não conheço suficiente sobre & 0 & $0[0 ; 0]$ & 5 & $6,7[2,6 ; 14]$ & 2 & $2,6[0,5 ; 8]$ & 0,150 \\
\hline
\end{tabular}

Modelo Equações de Estimações Generalizadas; Letras distintas representam categorias com distribuições estatisticamente distintas. 
Para a avaliação de desempenho dos estudantes foi citada a utilização de mais de um instrumento (média de 3,1 $\pm 1,7$ ), sendo os mais utilizados "relatório feito pelo estudante sobre as atividades desenvolvidas no estágio" (80,6\%; $\mathrm{n}=79)$, "feedback feito pelo supervisor sobre o desempenho do estudante" (64,3\%; $\mathrm{n}=63)$ e "autoavaliação" $(34,7 \%$; $\mathrm{n}=31)$. O instrumento utilizado em maior proporção sozinho para avaliação de desempenho do estudante foi o "relatório oral elou escrito das atividades desenvolvidas no estágio" $(11,2 \% ; \mathrm{n}=11)$. Na Tabela 6 encontra-se descrita a proporção de cursos de Farmácias que utilizavam cada instrumento.

\begin{tabular}{lc} 
Tabela 6: Instrumentos para avaliação dos estudantes nos estágios em farmácias comunitárias (n \\
\multicolumn{1}{c}{ Instrumentos de avaliação } & $\mathbf{n}(\%)$ \\
\hline $\begin{array}{l}\text { Relatório oral e/ou escrito das atividades desenvolvidas no estágio } \\
\text { Feedback oral ou escrito, feito pelo supervisor, sobre a performance do }\end{array}$ & $79(80,6)$ \\
estudante & $63(64,3)$ \\
Autoavaliação do estudante & $34(34,7)$ \\
Portfólio & $31(31,6)$ \\
Prova escrita & $23(23,5)$ \\
Longbook & $20(20,4)$ \\
Discussão nos Fóruns, utilizando o Ambiente Virtual de Aprendizagem & $13(13,3)$ \\
Exame Clínico Objetivo Estruturado (OSCE) & $11(11,2)$ \\
Long-case & $11(11,2)$ \\
Mini-Cex & $11(11,2)$ \\
MiniPat & $5(5,1)$ \\
Patient-survey (PS) & $3(3,1)$ \\
Não existe nenhum processo de avaliação & $1(1)$ \\
\hline
\end{tabular}

\section{DISCUSSÃO}

A transformação dos cenários de prática em espaços de ensino-aprendizagem requer a participação compartilhada de docentes, profissionais e acadêmicos, de modo que todos se sintam corresponsáveis pela articulação ensino-serviço [11]. No entanto, este estudo revela uma fragilidade na articulação entre a academia e o serviço, expressa na dificuldade de alocação dos estudantes nos serviços de saúde, frequência relativamente baixa dos docentes nos locais de prática e não inclusão de preceptores, na maioria dos cursos, no processo de planejamento dos estágios, sendo sua atribuição limitada apenas ao processo de supervisão e avaliação do estudante.

A literatura aponta que integração ensino-serviço-comunidade tem se configurado como importante estratégia de promoção de mudanças no ensino de graduação das diversas profissões da área da saúde no Brasil [8]. No entanto, os cursos de Farmácia têm apresentado dificuldades em inserir seus estudantes em farmácias comunitárias, principalmente naquelas vinculadas ao SUS. Essa problemática contrapõe-se às recomendações das DCN e dos Contratos Organizativos de Ação Pública Ensino-Saúde (COAPES), os quais visam fortalecer o processo de integração ensinoserviço-comunidade para os cursos da área da saúde, garantindo o acesso a todos os estabelecimentos de saúde, sob a responsabilidade do gestor, como cenário de práticas para a formação no âmbito da graduação e da residência em saúde [10, 27].

Diversos fatores podem estar contribuindo com essa problemática: o crescimento exponencial dos cursos de Farmácia no Brasil, que tem gerado competição pelos locais de estágio [28, 29]; a 
abordagem tradicional, ainda prevalente nos cursos de Farmácia, a qual separa a teoria da prática, transferindo aos preceptores o papel de ensinar os estudantes a aplicar o conhecimento na prática profissional, o que gera sobrecarga a esse profissional [4]; e a carga horária baixa para os estágios em farmácias comunitárias, em relação aos $20 \%$ das 4000 horas mínimos exigidos nas DCN, que dificulta aos estudantes assumirem responsabilidades crescentes ao longo do curso nos locais de prática e ao final se tornarem independentes de forma a contribuir com o serviço de modo mais enfático e sistemático.

Um ponto nevrálgico nessa articulação diz respeito ao papel do docente e preceptor no processo de ensino aprendizagem nos estágios. Os dados apontam que os docentes, na maioria dos cursos participantes, vão aos locais de prática com pouca frequência em relação aos encargos docentes atribuídos para a orientação e à carga horária que o estudante está em campo. Assim, o preceptor possui um papel chave, uma vez que cabe a ele integrar os valores e conceitos acadêmicos no mundo do trabalho. Contudo isso requer sensibilização e qualificação clínica e pedagógica [30, 31]. A formação de preceptores é um dos pilares para o sucesso da aprendizagem clínica de estudantes de Farmácia [7, 32-34] e o docente deve contribuir no processo de qualificação e educação continuada desses profissionais, com vistas à melhoria do serviço e do processo de ensinoaprendizagem nos cenários de práticas [10, 27]. No entanto, esta não é uma realidade nos cursos de Farmácia no Brasil.

No Brasil, mesmo após os avanços na formação e regulamentação das atribuições clínicas [3538], o quantitativo de farmacêuticos com conhecimento e habilidades adequados para essa prática é muito pequeno e os serviços farmacêuticos ainda se encontram desarticulados das necessidades dos pacientes e dos demais serviços de saúde [32, 39-41]. A integração ensino-serviço-comunidade propicia o encontro de usuários, trabalhadores, gestores, discentes e docentes; modifica as práticas de saúde e de educação na comunidade e colabora para a formação e a educação permanente de profissionais, em consonância com as necessidades do sistema de saúde [19]. Dessa forma, a integração academia-serviço se constitui como um potencial para promover mudanças nesse cenário da atuação clínica dos farmacêuticos.

Todavia, a falta de comprometimento com as atribuições previstas para os atores envolvidos no processo de integração-ensino-serviço-comunidade tem tornado as farmácias universitárias locais estratégicos no processo de ensino-aprendizagem para o cuidado em saúde, uma vez que neste cenário os estudantes têm maior probabilidade de estarem expostos e experienciar os serviços de cuidado farmacêutico, além do acesso ser mais facilitado.

De acordo com os princípios da aprendizagem experiencial, além do cenário de prática, para que a experiência vivenciada gere aprendizagem, há necessidade da articulação entre teoria e prática, da ação e reflexão. A partir da experiência concreta, há criação de hipóteses; posteriormente, a fim de solucioná-las, ocorre a conceituação abstrata, para a qual utiliza-se da lógica e desenvolvimento de princípios teóricos; e, finalmente, a experimentação ativa, que resultará em novas experiências [2, 42]. Assim, a inserção de conteúdo das "ciências sociais/comportamentais/administrativas de farmácia" e de "ciências clínicas", não observada na maioria dos cursos de Farmácia participantes, são imprescindíveis antes da inserção do estudante nos estágios em farmácia comunitária. Essa ausência prejudica o processo de ensino-aprendizagem, uma vez que impossibilita que o estudante acesse conteúdos, o que compromete o desenvolvimento de competências intermediárias e finais necessárias para o cuidado em saúde [19].

No Brasil, houve importantes avanços no que se refere à incorporação de conteúdo das ciências clínicas e, em menor proporção, das ciências humanas e sociais nos currículos dos cursos de Farmácia, porém esse processo ocorreu numa lógica disciplinar [43]. Isso dificulta a recuperação de conhecimentos para aplicá-los na prática do cuidado ao paciente, causando uma lacuna entre "o saber e o fazer" [44].

Além de conteúdos mínimos necessários para a prática profissional, a metodologia de ensinoaprendizagem utilizada nos estágios deve propiciar que o estudante desenvolva autonomia profissional e, para isso, os estágios devem ser pensados de modo a promover o desenvolvimento crescente das competências e responsabilidades, como relatado pela maioria dos participantes estudante observa e depois executa com supervisão". Kolb (2015) [2] destaca que a "aprendizagem é um processo contínuo e ascendente impulsionado pela experiência", sendo que a transição entre os níveis de aprendizagem (aquisitivo, especializado e integrativo) depende da apropriação das 
experiências culturais que cada pessoa em particular vivência [42]. Desse modo, o método de ensino-aprendizagem utilizado nos estágios deve ser cuidadosamente planejado pelo docente e preceptor, no momento da inserção do estudante em contato direto com o paciente, de modo a não expor o paciente a riscos.

Dessa maneira, a avaliação deve ser parte integrante de todo processo educacional, do início ao fim, sendo que para ter impacto educacional, além de válida, precisa produzir reflexão do estudante sobre a sua prática e permiti-lo criar consciência sobre as necessidades de aprendizado com o objetivo de melhorar seu desempenho futuro. Isso é possível por meio de um feedback efetivo do docente, ou seja, a avaliação formativa é um elemento imprescindível para melhorar a aprendizagem em curso [45]. No entanto, com a presença pouco frequente dos docentes nos locais de estágio isso dificulta um feedback efetivo e, dessa forma, a avaliação formativa, ficando essa função, provavelmente, a cargo do preceptor, reforçando novamente a importância de qualificá-lo.

A escolha do instrumento para mensurar o desempenho dos estudantes deve considerar a metodologia de ensino-aprendizagem e objetivos educacionais propostos. No ensino experiencial, o componente "fazer" é o mais desafiador de ser avaliado, não podendo ser mensurado por meio de métodos tradicionais, como prova escrita, pois estes mensuram apenas o conhecimento teórico [46]. Os cursos de Farmácia, em sua maioria, têm utilizado instrumentos que não possuem capacidade de mensurar desempenho, como relatório de atividades realizadas e avaliação subjetiva dos supervisores. No entanto, havia IES que agregava a autoavaliação e o portfólio como métodos de avaliação, e outras, em menor proporção, instrumentos como OSCE, Mini-Cex e Long-Case.

Segundo Tibério et al. (2012) [45], estes instrumentos tem sido utilizados nos cursos de Medicina, pois garantem validade na avaliação de habilidades clínicas por permitirem autenticidade, sendo o OSCE utilizado em ambientes simulados, e o Long-case e Mini-CEX na avaliação em ambientes reais. Alguns pesquisadores defendem a utilização de instrumentos de avaliação baseados no princípio da objetividade [3]. Em contraste, Kolb (2015) [2] entende que a "avaliação da aprendizagem deve ocorrer pelos processos e não pelos produtos", uma vez que conhecer é um processo de contínua invenção e recriação hipotética sobre a realidade. Sob essa perspectiva, o portfólio pode ser um instrumento interessante, pois possibilita a avaliação do processo de aprendizagem do estudante [45]. Nos Estados Unidos, o portfólio é bastante utilizado no currículo experiencial como forma de avaliação formativa, no entanto, pesquisadores sugerem a necessidade de mais estudos para o seu uso na avaliação somativa $[47,48]$. No Brasil, tem sido utilizado, principalmente, em programas de integração ensino-serviço-comunidade, uma vez que permitem que se registre o "caminho cognitivo" do estudante em áreas de difícil avaliação, tais como profissionalismo e atitude [45].

Como limitação do estudo destaca-se a dificuldade de contatar os docentes responsáveis pelos estágios em farmácia comunitária de todos os cursos de Farmácia consideráveis elegíveis para o estudo. Além disso, cabe destacar que um número expressivo, mesmo após apresentar o trabalho e se comprometer a participar, não responderam ao questionário. Outra limitação, própria dos estudos quantitativos, refere-se à impossibilidade de determinar todas as configurações possíveis dos modelos de ensino-aprendizagem e avaliação.

\section{CONCLUSÃO}

Diante desses resultados, os cursos de graduação em Farmácia no Brasil devem atentar-se na construção de programas de ensino-aprendizagem nos estágios em farmácia comunitária. Isso pode ser justificado, principalmente, pelo baixo quantitativo de horas em que os alunos são expostos a ambientes de prática, a desarticulação entre academia e serviços de saúde expresso pela dificuldade em alocar estudantes, falta de preocupação com a qualificação dos preceptores e, consequentemente, com o processo de ensino-aprendizagem, e fragilidade no processo de avaliação do estudante.

É necessário investir em parcerias com o serviço de saúde, em que ambos se sintam corresponsáveis pela formação de profissionais e estruturar programas de estágios, pautados nas particularidades e necessidades do sistema de saúde brasileiro e na formação de farmacêuticos condizente com as necessidades da população. Isso requer qualificação clínica e pedagógica dos 
preceptores para que a experiência resulte em aprendizagem e transforme os estudantes em profissionais críticos e qualificados.

A efetivação da integração-ensino-serviço-comunidade é uma estratégia, que deve ser fortalecida nos cursos de Farmácia, pois permite o ensino-aprendizagem dos futuros farmacêuticos, em um cenário de prática real e interdisciplinar, e garante a qualificação profissional em consonância com as necessidades do sistema de saúde, contribuindo para a formação de farmacêuticos com competências para atuar no cuidado em saúde. Estudos mais aprofundados são imprescindíveis para a compreensão do processo de ensino-aprendizagem que resulta em competências para o cuidado em saúde.

\section{AgRADECIMENTOS}

Os autores gostariam de agradecer ao Sr. Luciano Santos Pinto Guimarães por auxiliar na análise estatística.

\section{REFERÊNCIAS BIBLIOGRÁFICAS}

1. Kassam R, Kwong M, Collins JB. Promoting direct patient care services at community pharmacies through advanced pharmacy practice experiences. Pharm Prat. 2013;(21):368-77, doi: 10.1111/ijpp.12016.

2. Kolb DA. Experiential learning: Experience as the source of learning and development. 2nd ed. New Jersey (US): Pearson Education Inc; 2015. 390 p.

3. Losinski V. Educating for action: understanding the development of pharmaceutical care practitioners [master's dissertation]. Minesota (US): University of Minnesota; 2011. $247 \mathrm{p}$.

4. Turner CJ, Ellis S, Giles J, Maffeo C, Hansen L, Saseen JJ, et al. A strategy to develop advanced pharmacy practice experiences. Am J Pharm Educ. 2007;71(3):1-8, doi: 10.5688/aj710346.

5. de Freitas EL, de Oliveira DR. Critical thinking in the context of clinical practice : The need to reinvent pharmacy education. Rev Port Educ. 2015;28(2):231-50, doi: 10.21814/rpe.7753.

6. Austin Z, Ensom MHH. Education of pharmacists in Canada. Am J Pharm Educ. 2008;72(6):1-11, doi: $10.5688 / a j 7206128$.

7. Danielson J, Craddick K, Eccles D, Kwasnik A, Sullivan TAO. A qualitative analysis of common concerns about challenges facing pharmacy experiential education programs. Am J Pharm Educ. 2015;79(1):1-8, doi: 10.5688/ajpe79106.

8. Albuquerque VS, Gomes AP, de Rezende CHA, Sampaio MX, Dias OV, Lugarinho RM. A integração ensino-serviço no contexto dos processos de mudança na formação superior dos profissionais da saúde. Rev Bras Educ Med. 2008;32(3):356-62, doi: 10.1590/S0100-55022008000300010.

9. Conselho Nacional de Educação (BR). Parecer CNE/CES n 1.300/2001, de 6 de novembro de 2001. Institui as Diretrizes Curriculares Nacionais dos cursos de graduação em Farmácia e Odontologia. Diário Oficial da União. 7 dez 2001;223(Seção 1):25.

10. Conselho Nacional de Educação (BR). Resolução no 6, de 19 de outubro de 2017. Institui as Diretrizes Curriculares Nacionais do curso de graduação em Farmácia e dá outras providências. Diário Oficial da União. 20 out 2017;202(Seção 1):30-2.

11. Gonçalves CNS, Corrêa AB, Simon G, do Prado ML, Rodrigues J, Reibnitz KS. Integração ensino-serviço na voz de profissionais de saúde. Rev Enferm UFPE. 2014;8(6):1678-86, doi: 10.5205/reuol.5876-506101-SM.0806201430.

12. Brasil, Conselho Nacional de Educação. Lei no ${ }^{\circ} 11.788$, de 25 de setembro de 2008. Dispõe sobre o estágio de estudantes; (...) e dá outras providências. Diário Oficial da União. 26 set 2008;187(Seção 1):3-4.

13. Jabbur-Lopes MO, Mesquita AR, Silva LMA, de Almeida-Neto A, Lyra-Junior DP. Virtual patients in pharmacy education. Am J Pharm Educ. 2012;76(5):1-8, doi:10.5688/ajpe76592.

14. Mesquita AR, Souza WM, Boaventura TC, Barros IMC, Antoniolli AR, Silva WB, et al. The effect of active learning methodologies on the teaching of pharmaceutical care in a Brazilian pharmacy faculty. PLoS One. 2015;10(5):1-16, doi: 10.1371/journal.pone.0123141.

15. de Souza WM, Mesquita AR, Antoniolli AR, de Lyra-Junior DP, da Silva WB. Teaching in pharmaceutical care: A systematic review. African J Pharm Pharmacol. 2015;9(10):321-6, doi: 10.5897/AJPP2014. 4181.

16. Mendonça SDAM, de Freitas EL, de Oliveira DR. Competencies for the provision of comprehensive medication management services in an experiential learning project. PLoS One. 2017;12(9):1-14, doi: 10.1371/journal.pone. 0185415 . 
17. de Carvalho MN, Leite SN. Mercado de trabalho farmacêutico no Brasil: 2010 a 2015. 1st ed. São Paulo: Escola Nacional dos Farmacêuticos; 2016. 32 p.

18. International Pharmaceutical Federation. A global competency framework for services provided by pharmacy workforce [Internet]. 2012 [acesso em 21 fev 2020]. 21 p. Disponível em: https://www.fip.org/files/fip/PharmacyEducation/GbCF_v1.pdf

19. Conselho Federal de Farmácia. Competências para a atuação clínica do farmacêutico: Relatório do I Encontro Nacional de Educadores em Farmácia Clínica e Matriz de Competências para a Atuação Clínica. Brasília (DF): CFF; 2017. 124 p.

20. Brasil, Ministério da Educação. Cadastro Nacional de Cursos e Instituições de Educação Superior Cadastro e-MEC. c2021 [acesso em 2021]. Disponível em: https://emec.mec.gov.br

21. Conselho Federal de Farmácia. Serviços farmacêuticos diretamente destinados ao paciente, família e à comunidade: Contextualização e arcabouço conceitual. Brasília (DF): CFF; 2016. 200 p.

22. Accreditation Council for Pharmacy Education. Accreditation standards and key elements for the professional program in pharmacy leading to the doctor of Pharmacy degree ("STANDARDS 2016"). Illinois (US): ACPE; 2015. 31 p.

23. Instituto Regional Faimer Brasil. Glossário de métodos de avaliação dos estudantes. [local desconhecido: editora desconhecida]; 2016. 4 p.

24.Zeger SL, Liang K-Y. Longitudinal data analysis for discrete and continuous outcomes. Biometric. 1986;42(1):121-30, doi: 10.2307/2531248.

25. Liang K-Y, Zeger SL. Longitudinal data analysis using generalized linear models. Biometric. 1986;73(1):13-22, doi: 10.1093/biomet/73.1.13.

26. Guimarães LSP, Hirakata VN. Use of the generalized estimating equation model in longitudinal data analysis. Rev HCPA. 2012;32(4):503-11.

27. Brasil. Portaria Interministerial n ${ }^{\circ} 1.127$, de 04 de agosto de 2015. Institui as diretrizes para a celebração dos Contratos Organizativos de Ação Pública Ensino-Saúde (COAPES), para o fortalecimento da integração entre ensino, serviços e comunidade no âmbito do SUS. Diário Oficial da União. 5 ago 2015;148(Seção1):193-6.

28. Kennerly J, Weber RJ. Role of pharmacy education in growing the pharmacy practice model. Hosp Pharm. 2013;48(4):338-42, doi: 10.1310/hpj4804-338.

29. Lopes NMS, Gondim APS, Soares ACS, dos Santos DB, de Sales-Neto MR, Pinto DM. A quantitative analysis of the quality of pharmacy education in Brazil. Am J Pharm Educ. 2019;83(3):291-7, doi: 10.5688/ajpe6543.

30. Finkler RU, da Silva AS, Bonamigo AW. Visão dos preceptores quanto à preceptoria e o acolhimento do estudante de graduação na atenção primária à saúde. Res Soc Dev. 2019;8(2):1-17, doi: 10.33448/rsd$\mathrm{v} 8 \mathrm{i} 2.557$.

31. Botti SHO, Rego S. Preceptor, supervisor, tutor e mentor: Quais são seus papéis? Rev Bras Educ Med. 2008;32(3):363-73, doi: 10.1590/S0100-55022008000300011.

32. Baldoni ADO, Obreli-Neto PR, Guidoni CM, Pereira LRL. Perspectives for Clinical Pharmacy in Brazil. J Appl Pharm Sci. 2016;2(3):45-6.

33. Boyle CJ, Morgan JA, Layson-Wolf C, de Bittne MR. Developing and implementing an academy of preceptors. Am J Pharm Educ. 2009;73(2):1-10. doi: 10.5688/aj730234.

34. Vos SS, Trewet CB. A comprehensive approach to preceptor development. Am J Pharm Educ. 2012;76(3):1-7, doi: 10.5688/ajpe76347.

35. Brasil. Ministério da saúde. Diretrizes do NASF - Núcleo de Apoio a Saúde da Família. 1st ed. Brasília (DF): Ministério da Saúde; 2009. p. 164.

36. Conselho Federal de Farmácia. Resolução n 586 de 29 de agosto de 2013. Regula a prescrição farmacêutica e dá outras providências. Diário Oficial da União. 26 set 2013;187(Seção 1):136-8.

37. Conselho Federal de Farmácia. Resolução $n^{\circ}$ 585, de 29 de agosto de 2013. Regulamenta as atribuições clínicas do farmacêutico e dá outras providências. Diário Oficial da União. 25 set 2013;186(Seção 1):1868.

38. Conselho Federal de Farmácia. Resolução n ${ }^{\circ}$ 572, de 25 de abril de 2013. Dispõe sobre a regulamentação das especialidades farmacêuticas, por linhas de atuação. Diário Oficial da União. 06 mai 2013;85(Seção 1):143-4.

39. Nakamura CA, Leite SN. A construção do processo de trabalho no Núcleo de Apoio à Saúde da Família: a experiência dos farmacêuticos em um município do sul do Brasil. Cien Saude Colet. 2016;21(5):156572, doi: 10.1590/1413-81232015215.17412014.

40. Oliveira AB, Oyakawa CN, Miguel MD, Zanin SMW, Montrucchio DP. Obstáculos da atenção farmacêutica no Brasil. Braz J Pharm Sci. 2005;41(4):409-13, doi: 10.1590/S1516-93322005000400002.

41. Pereira LRL, de Freitas O. A evolução da Atenção Farmacêutica e a perspectiva para o Brasil. Braz J Pharm Sci. 2008;44(4):601-11, doi: 10.1590/S1516-93322008000400006. 
42. Pimentel A. A teoria da aprendizagem experiencial como alicerce de estudos sobre desenvolvimento profissional. Estud Psicol. 2007;12(2):159-68, doi: 10.1590/S1413-294X2007000200008.

43. de Morais DCM. Um estudo demográfico/cartográfico e análise das matrizes curriculares dos cursos de farmácia [tese]. Piracicaba (SP): Universidade Metodista de Piracicaba; 2015. 366 p.

44. Mendonça SAM, Meireles BL, de Freitas EL, de Oliveira DR. Pharmacy practice experiential programs in the context of clinical education. Int $\mathbf{J}$ Pharm Pharm Sci. 2017;9(2):35-41, doi: 10.22159/ijpps.2017v9i2.16247.

45. Tibério IFLC, Daud-Gallotti RM, Troncon LEA, Martins MA. Avaliação prática de habilidades clínicas. 1st ed. São Paulo: Atheneu; 2012. 316 p.

46. Miller GE. The assessment of clinical skills competence performanace. Acad Med. 1990;65(9):63-7, doi: 10.1097/00001888-199009000-00045.

47. Daugherty KK, Cumberland DM. The use of portfolios in US pharmacy schools. Am J Pharm Educ. 2018;82(3):240-52, doi: 10.5688/ajpe6239.

48. Skrabal MZ, Turner PD, Jones RM, Tilleman JA, Coover KL. Portfolio use and practices in US colleges and schools of pharmacy. Am J Pharm Educ. 2012;76(3):1-8, doi: 10.5688/ajpe76346. 